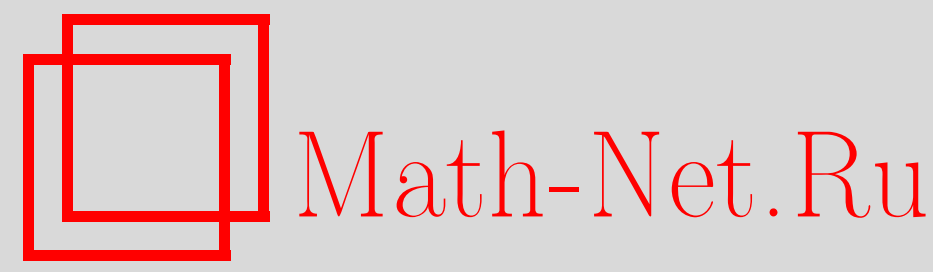

М. В. Медведева, Вложения классов непрерывных функций в классы функций ограниченной обобщенной вариации, Матем. сб., 2002, том 193, номер 7, 109-130

DOI: https://doi.org/10.4213/sm669

Использование Общероссийского математического портала Math-Net.Ru подразумевает, что вы прочитали и согласны с пользовательским соглашением http://www . mathnet.ru/rus/agreement

Параметры загрузки:

IP : 52.90 .164 .192

26 апреля 2023 г., $12: 45: 56$ 
УДК 517.518.2

\author{
М.В. Медведева
}

\title{
Вложения классов непрерывных функций в классы функций ограниченной обобщенной вариащии
}

\begin{abstract}
В работе исследуются необходимые и достаточные условия вложения классов функций $H^{\omega}$ в классы функций ограниченной обобщенной вариации. Получены теоремы как общего характера, так и теоремы вложения при некоторых дополнительных условиях, наложенных на модуль непрерьвности.

Библиография: 15 названий.
\end{abstract}

\section{Введение}

В теории функций действительного переменного встречаются различные обобщения понятия вариации функции. Некоторые из них появились в работах, где обобшалась теорема Жордана о сходимости тригонометрического ряда Фурье [1]. Различные обобщения этой теоремы на классы функций ограниченной $\Phi$-вариации получали Н. Винер [2], Дж. Марцинкевич [3], Л. Юнг, который ввел общее определение $\Phi$-вариации [4], Р. Салем [5]. Функции ограниченной $\Lambda$-вариации впервые были рассмотрены Д. Ватерманом в работе [6], где для них было получено обобщение теоремы Жордана о сходимости тригонометрического ряда Фурье. В частности, из результата Ватермана следуют все перечисленные выше теоремы. В дальнейшем исследовались и другие свойства функций ограниченной $\Lambda$-вариации.

ОПРЕДЕЛЕНИЕ 1 . Пусть $\Lambda=\left\{\lambda_{k}\right\}_{k=1}^{\infty}-$ монотонная последовательность положительных чисел такая, что $\lambda_{k} \rightarrow \infty$ при $k \rightarrow \infty$ и $\sum_{k=1}^{\infty}\left(1 / \lambda_{k}\right)=\infty$. Функция $f:[a, b] \rightarrow \mathbb{R}$ называется функцией с ограниченной $\Lambda$-вариачией $(f \in \Lambda B V)$, если

$$
\sup _{G} \sum_{k} \frac{\left|f\left(b_{k}\right)-f\left(a_{k}\right)\right|}{\lambda_{k}}<\infty
$$

где супремум берется по всем конечным системам $G$ попарно непересекающихся интервалов $\left(a_{k}, b_{k}\right)$ из отрезка $[a, b]$.

Далее для упрощения записи в качестве отрезка $[a, b]$ будем рассматривать отрезок $[0,1]$.

ОПРЕДЕЛЕниЕ 2. Функция $\omega$, определенная на $[0, \infty)$ или на $[0, l], 0<l<\infty$, называется модулем непрерывности, если она непрерьвна, не убывает, $\omega(0)=0$ и $\omega\left(t_{1}+t_{2}\right) \leqslant \omega\left(t_{1}\right)+\omega\left(t_{2}\right)$ при $t_{1}$ и $t_{2}$, для которых обе части неравенства имеют смысл.

Работа вьполнена при поддержке Российского фонда фундаментальных исследований (грант № 99-01-0062) и Программы поддержки ведущих научных школ (грант № 00-15-96143).

$$
\text { (C) M. B. Медведева } 2002
$$


ОПРЕДЕЛЕНИЕ 3 . Пусть на отрезке [0, 1] задан модуль непрерывности $\omega$. Через $H^{\omega}$ обозначим множество непрерывных на $[0,1]$ функций $f$, для которых $\omega(\delta, f)=$ $O(\omega(\delta))$ при $\delta \rightarrow 0$, где

$$
\omega(\delta, f)=\sup _{\substack{0 \leqslant h \leqslant \delta \\ 0 \leqslant x \leqslant 1-h}}|f(x+h)-f(x)|, \quad 0 \leqslant \delta \leqslant 1 .
$$

В настоящей работе исследуются необходимые и достаточные условия вложения классов функций $H^{\omega}$ в классы функций $\Lambda B V$ и некоторые смежные вопросы. Получены теоремы как общего характера, так и теоремы вложения при дополнительных условиях, наложенных на модуль непрерывности $\omega(t)$.

В работе [7] доказана

Теорема А. Для вложения $H^{\omega} \subset \Lambda B V$ необходимо и достаточно, чтобь для всякой последовательности $\left\{t_{k}\right\}_{k=1}^{\infty}$ такой, что $t_{k} \geqslant 0, \sum_{k=1}^{\infty} t_{k} \leqslant 1$, ряд $\sum_{k=1}^{\infty}\left(\omega\left(t_{k}\right) / \lambda_{k}\right)$ сходился.

При доказательстве теоремы А [7] получено также утверждение, которое мы сформулируем в теореме В и которое понадобится в дальнейшем. Обозначим через $T$ множество всех последовательностей $\left\{t_{k}\right\}_{k=1}^{\infty}, t_{k} \geqslant 0, \sum_{k=1}^{\infty} t_{k} \leqslant 1$.

Теорема В. Для вложения $H^{\omega} \subset \Lambda B V$ необходимо и достаточно, чтоби выполнялось условие $\sup _{T} \sum_{k=1}^{\infty}\left(\omega\left(t_{k}\right) / \lambda_{k}\right)<\infty$.

Теоремы А и В дают полное решение проблемы вложения $H^{\omega} \subset \Lambda B V$ в том смысле, что они содержат необходимое и достаточное условие вложения без каких-либо дополнительных предположений о модуле непрерывности и последовательности $\Lambda=\left\{\lambda_{k}\right\}_{k=1}^{\infty}$. Столь же полное решение получено А. С. Беловым [8], но его необходимое и достаточное условие вложения сильно отличается от условия в теореме А и в настоящей работе не используется. Вложения классов $H^{\omega}$ в классы функций ограниченной обобщенной вариации изучались также Е. И. Бережньм [9] и Л. Лейндлером [10], который обобщил теорему А на некоторые другие классы функций.

Насколько трудно или легко проверять условия вложения в теоремах $\mathrm{A}$ и $\mathrm{B}$, можно оценить только при рассмотрении частных случаев. Если $H^{\omega}$ является классом Липшица $\operatorname{Lip} \alpha, 0<\alpha<1$, то, полагая $\omega(t)=t^{1 / p}, p=1 / \alpha$, и применяя неравенство Гёльдера, из теоремы В получаем, что для вложения $\operatorname{Lip}(1 / p) \subset \Lambda B V$ необходимо и достаточно, чтобы $\sum_{k=1}^{\infty}\left(1 / \lambda_{k}^{q}\right)<\infty$, где $1 / p+1 / q=1$.

В более сложных частных случаях возникают трудности из-за необходимости рассматривать обширное семейство последовательностей $\left\{t_{k}\right\}_{k=1}^{\infty}$. Доказанная ниже теорема 1 позволяет делать вывод о вложении $H^{\omega} \subset \Lambda B V$, рассматривая лишь однопараметрическое семейство последовательностей $\left\{t_{k}\right\}_{k=1}^{\infty}$, для которых условие $\sum_{k=1}^{\infty} t_{k} \leqslant 1$ может не выполняться. На основании теорем 1, А и В нами получен ряд результатов при дополнительных предположениях о модуле непрерывности $\omega$. Эти результаты опубликованы без доказательства в [11]. В работе [11] рассматриваются классы $H^{\omega}$, в определенном смысле близкие к классам Липшица, и некоторые классы, содержащие объединение всех классов Липшица. Для классов, близких к $\operatorname{Lip}(1 / p), p>1$, вопрос о вложении $H^{\omega} \subset \Lambda B V$ сводится к 
изучению сходимости в точности одного числового ряда. Объем настоящей статьи не позволяет изложить доказательства всех теорем из работы [11]. Мы ограничимся лишш случаем классов $H^{\omega}$, промежуточных между Lip 1 и всеми другими классами Липшица. Точное определение рассматриваемых классов содержится в 1 . Именно для этого случая доказательства оказались наиболее трудоемкими. При этом условие вложения $H^{\omega} \subset \Lambda B V$ сводится к изучению сходимости однопараметрического семейства рядов, построение которых проше, чем в случае непосредственного использования теоремы 1.

\section{§1. Формулировки теорем}

С.Б. Стечкиным доказано [12], что для каждого модуля непрерывности $\omega$ существует вогнутый модуль непрерьвности $\omega^{*}$ такой, что $\omega(t) \leqslant \omega^{*}(t) \leqslant 2 \omega(t)$ при $t \in[0,1]$. Тогда класс $H^{\omega}$ совпадает с классом $H^{\omega^{*}}$. Ниже всюду предполагается, что $\omega$ - вогнутая функция $\left(\omega\left(\alpha t_{1}+(1-\alpha) t_{2}\right) \geqslant \alpha \omega\left(t_{1}\right)+(1-\alpha) \omega\left(t_{2}\right)\right.$, $\left.0 \leqslant t_{1}, t_{2} \leqslant 1,0 \leqslant \alpha \leqslant 1\right)$. Положив $\omega(t)=\omega(1)$ для $t \in(1, \infty)$, будем считать, что функция $\omega$ задана на $[0, \infty)$. Поскольку $\omega$ - вогнутая функция на $[0, \infty)$, то у нее всюду на $(0, \infty)$ сушествуют конечные правая производная $\omega_{+}^{\prime}(t)$ и левая производная $\omega_{-}^{\prime}(t)$, причем $\omega_{+}^{\prime}(t) \leqslant \omega_{-}^{\prime}(t)$ и $\omega_{+}^{\prime}(\tau) \geqslant \omega_{-}^{\prime}(t)$ для $\tau<t$. В точке $t=0$ правая производная $\omega_{+}^{\prime}(0)$ конечна или бесконечна. Обе односторонние производные - невозрастающие функции, равные нулю при $t>1$.

Если $\omega_{+}^{\prime}(0)<\infty$, то из определений классов $H^{\omega}$ и $\Lambda B V$ непосредственно следует, что $H^{\omega} \subset \Lambda B V$. В случае $\omega_{+}^{\prime}(0)=\infty$ введем функцию $t=t(\lambda)$ следующим образом. Для каждого $\lambda>0$ найдется $t \in(0, \infty)$ такое, что $\omega_{+}^{\prime}(t) \leqslant \lambda \leqslant \omega_{-}^{\prime}(t)$. Если таких значений $t$ больше одного (например, на промежутке, где $\omega^{\prime}(t)=$ const), то выберем одно из них и обозначим $t(\lambda)$. Таким образом,

$$
\omega_{+}^{\prime}(t(\lambda)) \leqslant \lambda \leqslant \omega_{-}^{\prime}(t(\lambda)) .
$$

Очевидно, что $t(\lambda)$ - невозрастающая функция и $\lim _{\lambda \rightarrow \infty} t(\lambda)=0$. Окрестностью нуля будем называть правую окрестность нуля, исключая нуль.

Teорема 1. Пусть $\lim _{t \rightarrow 0}(\omega(t) / t)=\infty$. Тогда для вложсения $H^{\omega} \subset \Lambda B V$ необходимо и достаточно, чтобь существовало число $c>0$, при котором ряд $\sum_{k=1}^{\infty}\left(\omega\left(t\left(c \lambda_{k}\right)\right) / \lambda_{k}\right)$ сходится.

Теоремы 2-4 потребуются при выводе условий вложения $H^{\omega} \subset \Lambda B V$ для классов, промежуточных между Lip 1 и другими классами Липшица. Правая производная в теореме 3 выбрана для определенности. Теорема остается верной, если $\omega_{+}^{\prime}(t)$ заменить на $\omega_{-}^{\prime}(t)$.

ОПРЕДЕЛЕНИЕ 4. Будем говорить, что модуль непрерывности $\omega(t)$ yдовлетворяет т-условию, если $\underline{\lim }_{t \rightarrow 0}\left(t \omega_{+}^{\prime}(t) / \omega(t)\right)>0$.

Это условие аналогично $\Delta_{2}$-условию для выпуклых функций [13].

ТЕОрема 2. Пусть $\lim _{t \rightarrow 0}(\omega(t) / t)=\infty u \omega(t)$ удовлетворяет m-условию. Тогда для вложения $H^{\omega} \subset \Lambda B V$ необходимо и достаточно, чтобь существовало число с $>0$, при котором ряд $\sum_{k=1}^{\infty} t\left(c \lambda_{k}\right)$ сходится. 
ТеОрема 3. Пусть на $(0, \infty)$ заданы функиии $G_{1}(z)$ и $G_{2}(z)$, положительнье и невозрастающие. Пусть также $\omega(t)$ удовлетворяет т-условию. Тогда

1) если в окрестности нуля выполняется неравенство $G_{1}\left(\omega_{+}^{\prime}(t)\right) \leqslant t$, то для влохения $H^{\omega} \subset \Lambda B V$ необходимо, чтобь при некотором $c>0$ сходился ряд $\sum_{k=1}^{\infty} G_{1}\left(c \lambda_{k}\right)$;

2) если в окрестности нуля выполняется неравенство $t \leqslant G_{2}\left(\omega_{+}^{\prime}(t)\right)$, то для вложения $H^{\omega} \subset \Lambda B V$ достаточно, чтоби при некотором $c>0$ сходился ряд $\sum_{k=1}^{\infty} G_{2}\left(c \lambda_{k}\right)$.

Через $\ln _{k}(x)$ обозначим $k$-кратный логарифм $\ln (\ln \ldots(\ln x))$, а через $\exp _{k}(x)-$ $k$-кратную экспоненту $\exp (\exp \ldots(\exp x))$. Положим $\ln _{0} x=\exp _{0} x=x$.

Пусть $\left\{\xi_{k}\right\}_{k=1}^{\infty}-$ неубывающая последовательность положительных чисел, $m \in \mathbb{N}$. Обозначим

$$
\mu=\inf \left\{c: c>0, \sum_{k=1}^{\infty} \frac{1}{\exp _{m}\left(c \xi_{k}\right)}<\infty\right\}
$$

Если при всех $c>0$ ряд $\sum_{k=1}^{\infty}\left(1 / \exp _{m}\left(c \xi_{k}\right)\right)$ расходится, то положим $\mu=\infty$.

Теорема 4. Справедливо равенство $\mu=\overline{\lim }_{k \rightarrow \infty}\left(\ln _{m} k / \xi_{k}\right)$.

Перейдем к описанию тех классов, к которым относятся последующие теоремы. В теории функций действительного переменного встречается класс $H^{\omega}$, где $\omega(t) \sim t|\ln t|$ при $t \rightarrow 0$ [14]. Это частный случай классов $H^{\omega}$, для которых в окрестности нуля $\omega(t)=t\left(\ln _{m-1}|\ln t|\right)^{\beta}, m \geqslant 1, \beta>0$. Запишем последнее равенство в виде $\omega(t)=t u(x)$, где $x=\ln _{m-1}|\ln t|$ при малых $t, u(x)=x^{\beta}$. Заметим, что $x u^{\prime}(x) / u(x)=\beta$. Будем рассматривать классы $H^{\omega}$, которые близки к только что описанньм в следуюшем смысле. Пусть $\omega(t)=t u(x)$ в окрестности нуля переменной $t$, где $x=\ln _{m-1}|\ln t|, m \geqslant 1$. Функция $u(x)$ может отличаться от $x^{\beta}$. Будем предполагать, что

$$
\frac{x u_{-}^{\prime}(x)}{u(x)}=\beta+o(1) \quad \text { при } \quad x \rightarrow \infty, \quad \beta>0,
$$

где $u_{-}^{\prime}(x)$ - левая производная функции $u(x)$ по $x$. Для такого $\omega$ легко проверить, что $\operatorname{Lip} 1 \subset H^{\omega} \subset \operatorname{Lip} \alpha \forall \alpha \in(0,1)$.

ТЕОРемА 5. 1) Пусть для модуля непрерьвности $\omega(t)=t u(x)$, где $x=$ $\ln _{m-1}|\ln t|$ при мальх $t, m \geqslant 1$, выполняется условие

$$
\frac{x u_{-}^{\prime}(x)}{u(x)}=\beta+O\left(\frac{1}{\ln x}\right) \quad \text { npu } \quad x \rightarrow \infty, \quad \beta>0, \quad \beta \neq 1 .
$$

Тогда для вложсения $H^{\omega} \subset \Lambda B V$ необходимо и достаточно, чтобы при некотором $c>0$

$$
\sum_{k=1}^{\infty}\left(\exp _{m} \frac{c \lambda_{k}^{(1+\beta) / \beta}}{\left(u\left(\lambda_{k}\right)\right)^{1 / \beta}}\right)^{-1}<\infty
$$

2) Если $O(1 / \ln x)$ заменить на $O\left(\left(\ln _{r} x\right)^{\sigma} / \ln x\right)$ со сколь угодно больиим натуральным $r \geqslant 2$ и сколь угодно мальмм $\sigma>0$, то для кажсдого $\beta, \beta>0$, $\beta \neq 1$, указанное условие вложения $H^{\omega} \subset \Lambda B V$ теряет силу как в части необходимости, так и в части достаточности. 
ТЕОРема 6. 1) Пусть для модуля непрерывности $\omega(t)=t u(x)$, где $x=$ $\ln _{m-1}|\ln t|$ при мальх $t, m \geqslant 1$, выполняется условие

$$
\frac{x u_{-}^{\prime}(x)}{u(x)}=1+O\left(\frac{1}{\sqrt{\ln x}}\right) \quad \text { npu } \quad x \rightarrow \infty .
$$

Тогда для вложения $H^{\omega} \subset \Lambda B V$ необходимо и достаточно, чтобь при некотором $c>0$

$$
\sum_{k=1}^{\infty}\left(\exp _{m} \frac{c \lambda_{k}^{2}}{u\left(\lambda_{k}\right)}\right)^{-1}<\infty
$$

2) Если $O(1 / \sqrt{\ln x})$ заменить на $O\left(\left(\ln _{r} x\right)^{\sigma} / \sqrt{\ln x}\right)$ со сколь угодно больиим натуральным $r \geqslant 2$ и сколь угодно мальм $\sigma>0$, то указанное условие влохсения $H^{\omega} \subset \Lambda B V$ не будет необходимым.

3) Если

$$
\frac{x u_{-}^{\prime}(x)}{u(x)}-1=o(1) \quad \text { npu } \quad x \rightarrow \infty
$$

и эта разность не меняет знака при больиих $x$, то указанное условие вложсения $H^{\omega} \subset \Lambda B V$ является достаточным независимо от порядка малости этой разности.

СлЕДСТВИЕ 1. Ecлu $\omega(t) \sim t\left(\ln _{m-1}|\ln t|\right)^{\beta_{m}}\left(\ln _{m}|\ln t|\right)^{\beta_{m+1}} \cdots\left(\ln _{n-1}|\ln t|\right)^{\beta_{n}}$ при $t \rightarrow 0, \beta_{m}>0, n \geqslant m \geqslant 1, \beta_{i} \in \mathbb{R}, i=m+1, \ldots, n$, то для влоэсения $H^{\omega} \subset \Lambda B V$ необходимо и достаточно, чтобы существовало число $с>0$, при котором сходится ряд

$$
\sum_{k}\left(\exp _{m} \frac{c \lambda_{k}^{1 / \beta_{m}}}{\left(\ln \lambda_{k}\right)^{\beta_{m+1} / \beta_{m}} \cdots\left(\ln _{n-m} \lambda_{k}\right)^{\beta_{n} / \beta_{m}}}\right)^{-1}
$$

где сумма берется по тем $k$, для которых члены ряда имеют смысл.

Теоремы 5,6 и следствие 1 в сочетании с теоремой 4 приводят к теоремам 7,8 и следствию 2.

ТЕОРемА 7. 1) Пусть для модуля непрерьвности $\omega(t)=t u(x)$, где $x=$ $\ln _{m-1}|\ln t|$ при мальх $t, m \geqslant 1$, выполняется условие

$$
\frac{x u_{-}^{\prime}(x)}{u(x)}=\beta+O\left(\frac{1}{\ln x}\right) \quad \text { npu } \quad x \rightarrow \infty, \quad \beta>0, \quad \beta \neq 1 .
$$

Тогда для вложения $H^{\omega} \subset \Lambda B V$ необходимо и достаточно, чтобъ

$$
\varlimsup_{k \rightarrow \infty} \frac{\left(\ln _{m} k\right)\left(u\left(\lambda_{k}\right)\right)^{1 / \beta}}{\lambda_{k}^{(1+\beta) / \beta}}<\infty
$$

2) Если $O(1 / \ln x)$ заменить на $O\left(\left(\ln _{r} x\right)^{\sigma} / \ln x\right)$ со сколь угодно больиим натуральным $r \geqslant 2$ и сколь угодно мальмм $\sigma>0$, то для кажсдого $\beta, \beta>0$, $\beta \neq 1$, указанное условие вложсения $H^{\omega} \subset \Lambda B V$ теряет силу как в части необходимости, так и в части достаточности. 
ТЕОРема 8. 1) Пусть для модуля непрерьвности $\omega(t)=t u(x)$, где $x=$ $\ln _{m-1}|\ln t|$ при мальх $t, m \geqslant 1$, выполняется условие

$$
\frac{x u_{-}^{\prime}(x)}{u(x)}=1+O\left(\frac{1}{\sqrt{\ln x}}\right) \quad \text { npu } \quad x \rightarrow \infty .
$$

Тогда для вложения $H^{\omega} \subset \Lambda B V$ необходимо и достаточно, чтобы

$$
\varlimsup_{k \rightarrow \infty} \frac{\left(\ln _{m} k\right) u\left(\lambda_{k}\right)}{\lambda_{k}^{2}}<\infty .
$$

2) Если $O(1 / \sqrt{\ln x})$ заменить на $O\left(\left(\ln _{r} x\right)^{\sigma} / \sqrt{\ln x}\right)$ со сколь угодно больиим натуральным $r \geqslant 2$ и сколь угодно мальм $\sigma>0$, то указанное условие влохсения $H^{\omega} \subset \Lambda B V$ не будет необходимым.

3) Если функиия $x u_{-}^{\prime}(x) / u(x)-1$ есть бесконечно малая при $x \rightarrow \infty$ ине меняет знака при больших $x$, то указанное условие вложсения $H^{\omega} \subset \Lambda B V$ является достаточным независимо от порядка малости этой функиии.

СлеДСТВИЕ 2. Eсли $\omega(t) \sim t\left(\ln _{m-1}|\ln t|\right)^{\beta_{m}}\left(\ln _{m}|\ln t|\right)^{\beta_{m+1}} \cdots\left(\ln _{n-1}|\ln t|\right)^{\beta_{n}}$ при $t \rightarrow 0, \beta_{m}>0, n \geqslant m \geqslant 1, \beta_{i} \in \mathbb{R}, i=m+1, \ldots, n$, то для вложсения $H^{\omega} \subset \Lambda B V$ необходимо и достаточно, чтобъ

$$
\varlimsup_{k \rightarrow \infty} \frac{\left(\ln _{m} k\right)\left(\ln \lambda_{k}\right)^{\beta_{m+1} / \beta_{m}} \cdots\left(\ln _{n-m} \lambda_{k}\right)^{\beta_{n} / \beta_{m}}}{\lambda_{k}^{1 / \beta_{m}}}<\infty .
$$

Мы оставляем теоремы 5 и 6 в качестве самостоятельных, так как в некоторых случаях их нетрудно применить, не обрашаясь к теореме 4. Например, если $\omega(t)=$ $t|\ln t|$ в окрестности нуля и $\lambda_{k}=k^{\alpha}, k \in \mathbb{N}, 0<\alpha \leqslant 1$.

\section{§2. Доказательства теорем}

Лемма 1. Пусть даны различные положсительные числа $M_{1}$ и $M_{2}$. Тогда

$$
\sup _{\sum_{k=1}^{\infty} \tau_{k} \leqslant M_{1}} \sum_{k=1}^{\infty} \frac{\omega\left(\tau_{k}\right)}{\lambda_{k}}, \quad \sup _{\sum_{k=1}^{\infty} \eta_{k} \leqslant M_{2}} \sum_{k=1}^{\infty} \frac{\omega\left(\eta_{k}\right)}{\lambda_{k}}
$$

либо оба конечны, либо оба равны $\infty$.

ДокАЗАТЕЛьство. Пусть, например, $M_{1}<M_{2}$. Ясно, что

$$
\sup _{\sum_{k=1}^{\infty} \tau_{k} \leqslant M_{1}} \sum_{k=1}^{\infty} \frac{\omega\left(\tau_{k}\right)}{\lambda_{k}} \leqslant \sup _{\sum_{k=1}^{\infty} \eta_{k} \leqslant M_{2}} \sum_{k=1}^{\infty} \frac{\omega\left(\eta_{k}\right)}{\lambda_{k}} .
$$

В силу вогнутости $\omega(t)$ для $M>1$ имеем $\omega(M t) \leqslant M \omega(t)$. Поэтому

$$
\begin{aligned}
& \sup _{\sum_{k=1}^{\infty} \eta_{k} \leqslant M_{2}} \sum_{k=1}^{\infty} \frac{\omega\left(\eta_{k}\right)}{\lambda_{k}}=\sup _{\sum_{k=1}^{\infty} \eta_{k}=M_{2}} \sum_{k=1}^{\infty} \frac{\omega\left(\frac{M_{2}}{M_{1}} \frac{M_{1} \eta_{k}}{M_{2}}\right)}{\lambda_{k}} \\
& \leqslant \sup _{\sum_{k=1}^{\infty} \eta_{k}=M_{2}} \frac{M_{2}}{M_{1}} \sum_{k=1}^{\infty} \frac{\omega\left(\frac{M_{1}}{M_{2}} \eta_{k}\right)}{\lambda_{k}}=\sup _{\sum_{k=1}^{\infty} \frac{M_{1}}{M_{2}} \eta_{k}=M_{1}} \frac{M_{2}}{M_{1}} \sum_{k=1}^{\infty} \frac{\omega\left(\frac{M_{1}}{M_{2}} \eta_{k}\right)}{\lambda_{k}} \\
& =\frac{M_{2}}{M_{1}} \sup _{\sum_{k=1}^{\infty} \tau_{k}=M_{1}} \sum_{k=1}^{\infty} \frac{\omega\left(\tau_{k}\right)}{\lambda_{k}} .
\end{aligned}
$$

Отсюда следует утверждение леммы. 
Лемма 2. Пусть $\lim _{t \rightarrow 0}(\omega(t) / t)=\infty$. Если при некотором $c>0$ ряд $\sum_{k=1}^{\infty}\left(\omega\left(t\left(c \lambda_{k}\right)\right) / \lambda_{k}\right)$ сходится, то ряд $\sum_{k=1}^{\infty} t\left(c \lambda_{k}\right)$ также сходится.

ДоКАЗАТЕльство. В силу вогнутости модуля непрерьвности $\omega(t)$ выполняется неравенство $\omega(t) / t \geqslant \omega_{-}^{\prime}(t)$. В частности, $\omega\left(t\left(c \lambda_{k}\right)\right) / t\left(c \lambda_{k}\right) \geqslant \omega_{-}^{\prime}\left(t\left(c \lambda_{k}\right)\right)$. $\mathrm{C}$ другой стороны, $c \lambda_{k} \leqslant \omega_{-}^{\prime}\left(t\left(c \lambda_{k}\right)\right)$ по определению функции $t(\lambda)$. Поэтому $\omega\left(t\left(c \lambda_{k}\right)\right) / t\left(c \lambda_{k}\right) \geqslant c \lambda_{k}$, а значит,

$$
t\left(c \lambda_{k}\right) \leqslant \frac{1}{c} \frac{\omega\left(t\left(c \lambda_{k}\right)\right)}{\lambda_{k}} .
$$

Отсюда следует утверждение леммы.

ДОКАЗАТЕЛЬСТво ТЕОРЕМЫ 1 . ДЛя краткости записи обозначим $t_{k}^{c}=t\left(c \lambda_{k}\right)$.

Достаточность. Пусть для некоторого $c>0$ ряд $\sum_{k=1}^{\infty}\left(\omega\left(t_{k}^{c}\right) / \lambda_{k}\right)$ сходится. Тогда по лемме 2 ряд $\sum_{k=1}^{\infty} t_{k}^{c}$ также сходится. Обозначим его сумму через $A$. Докажем, что

$$
\sup _{\sum_{k=1}^{\infty} t_{k} \leqslant A} \sum_{k=1}^{\infty} \frac{\omega\left(t_{k}\right)}{\lambda_{k}}=\sum_{k=1}^{\infty} \frac{\omega\left(t_{k}^{c}\right)}{\lambda_{k}} .
$$

В силу вогнутости $\omega(t)$ имеем для $\varepsilon>0$ и натурального числа $k$

$$
\omega\left(t_{k}^{c}+\varepsilon\right)-\omega\left(t_{k}^{c}\right) \leqslant \omega_{+}^{\prime}\left(t_{k}^{c}\right) \varepsilon \leqslant c \lambda_{k} \varepsilon, \omega\left(t_{k}^{c}\right)-\omega\left(t_{k}^{c}-\varepsilon\right) \geqslant \omega_{-}^{\prime}\left(t_{k}^{c}\right) \varepsilon \geqslant c \lambda_{k} \varepsilon .
$$

Тогда

$$
\frac{\omega\left(t_{k}^{c}+\varepsilon\right)-\omega\left(t_{k}^{c}\right)}{\lambda_{k}} \leqslant c \varepsilon, \quad \frac{\omega\left(t_{k}^{c}\right)-\omega\left(t_{k}^{c}-\varepsilon\right)}{\lambda_{k}} \geqslant c \varepsilon .
$$

Возьмем последовательность $\left\{t_{k}\right\}_{k=1}^{\infty}, \sum_{k=1}^{\infty} t_{k}=A$, отличную от $\left\{t_{k}^{c}\right\}_{k=1}^{\infty}$, и представим соответствующий ряд в виде

$$
\sum \frac{\omega\left(t_{n_{i}}^{c}+\varepsilon_{n_{i}}\right)}{\lambda_{n_{i}}}+\sum \frac{\omega\left(t_{m_{i}}^{c}-\varepsilon_{m_{i}}\right)}{\lambda_{m_{i}}}+\sum \frac{\omega\left(t_{p_{i}}^{c}\right)}{\lambda_{p_{i}}}
$$

где все $\varepsilon_{n_{i}}, \varepsilon_{m_{i}}>0$ и $\sum \varepsilon_{n_{i}}=\sum \varepsilon_{m_{i}}$. Составим и оценим разность

$$
\begin{aligned}
& \left(\sum \frac{\omega\left(t_{n_{i}}^{c}+\varepsilon_{n_{i}}\right)}{\lambda_{n_{i}}}+\sum \frac{\omega\left(t_{m_{i}}^{c}-\varepsilon_{m_{i}}\right)}{\lambda_{m_{i}}}+\sum \frac{\omega\left(t_{p_{i}}^{c}\right)}{\lambda_{p_{i}}}\right)-\sum_{k=1}^{\infty} \frac{\omega\left(t_{k}^{c}\right)}{\lambda_{k}} \\
& \quad=\sum \frac{\omega\left(t_{n_{i}}^{c}+\varepsilon_{n_{i}}\right)-\omega\left(t_{n_{i}}^{c}\right)}{\lambda_{n_{i}}}-\sum \frac{\omega\left(t_{m_{i}}^{c}\right)-\omega\left(t_{m_{i}}^{c}-\varepsilon_{m_{i}}\right)}{\lambda_{m_{i}}} \\
& \quad \leqslant \sum c \varepsilon_{n_{i}}-\sum c \varepsilon_{m_{i}}=c\left(\sum \varepsilon_{n_{i}}-\sum \varepsilon_{m_{i}}\right)=0 .
\end{aligned}
$$

Итак,

$$
\sup _{\sum_{k=1}^{\infty} t_{k} \leqslant A} \sum_{k=1}^{\infty} \frac{\omega\left(t_{k}\right)}{\lambda_{k}}=\sum_{k=1}^{\infty} \frac{\omega\left(t_{k}^{c}\right)}{\lambda_{k}} .
$$

Следовательно, $H^{\omega} \subset \Lambda B V$ по лемме 1 и теореме В. 
Необходимость. Предположим, что $H^{\omega} \subset \Lambda B V$, но $\sum_{k=1}^{\infty}\left(\omega\left(t_{k}^{c}\right) / \lambda_{k}\right)=\infty$ для всех $c>0$. Рассмотрим два случая.

Случай 1. Сушествует число $c>0$ такое, что $\sum_{k=1}^{\infty} t_{k}^{c}=A<\infty$. Тогда $\sup _{\sum_{k=1}^{\infty} t_{k} \leqslant A} \sum_{k=1}^{\infty}\left(\omega\left(t_{k}\right) / \lambda_{k}\right)=\infty$. В силу леммы 1 и теоремы В это противоречит вложению $H^{\omega} \subset \Lambda B V$.

Случай 2. Пусть $\sum_{k=1}^{\infty} t_{k}^{c}=\infty$ при всех $c>0$. В силу вогнутости $\omega$ выполняется неравенство $\omega(t) / t \geqslant \omega_{-}^{\prime}(t)$. Поэтому $\omega\left(t_{k}^{c}\right) / \lambda_{k} \geqslant c t_{k}^{c}$ при всех $c>0$ и натуральных $k$. Поскольку $\omega^{\prime}(t)=0$ на $(1, \infty)$, то все $t_{k}^{c} \in(0,1]$. При каждом фиксированном $c$ выберем точки $t_{1}^{c}, t_{2}^{c}, \ldots, t_{N_{c}}^{c}$ так, чтобы $1 \leqslant \sum_{k=1}^{N_{c}} t_{k}^{c} \leqslant 2$. Тогда $\sum_{k=1}^{N_{c}}\left(\omega\left(t_{k}^{c}\right) / \lambda_{k}\right) \geqslant \sum_{k=1}^{N_{c}} c t_{k}^{c} \geqslant c$. Следовательно, для всякого $c>0$ имеем $\sup _{\sum_{k=1}^{\infty}} t_{k} \leqslant 2 \sum_{k=1}^{\infty}\left(\omega\left(t_{k}\right) / \lambda_{k}\right) \geqslant c$ и, значит, $\sup _{\sum_{k=1}^{\infty} t_{k} \leqslant 2} \sum_{k=1}^{\infty}\left(\omega\left(t_{k}\right) / \lambda_{k}\right)=\infty$. В силу леммы 1 и теоремы В это противоречит вложению $H^{\omega} \subset \Lambda B V$. Теорема доказана.

ДоКАЗАТЕЛЬСТво ТЕоремы 2 . Так как $\omega(t)$ удовлетворяет $m$-условию, то найдется число $A>0$ такое, что при достаточно малых $t$ верно $t \omega_{+}^{\prime}(t) / \omega(t) \geqslant A$, т.е. $\omega(t) / \omega_{+}^{\prime}(t) \leqslant t / A$. Значит, для фиксированного $c>0$ и больших $\lambda$

$$
\frac{\omega(t(c \lambda))}{\lambda}=c \frac{\omega(t(c \lambda))}{c \lambda} \leqslant c \frac{\omega(t(c \lambda))}{\omega_{+}^{\prime}(t(c \lambda))} \leqslant c \frac{t(c \lambda)}{A} .
$$

Следовательно, из сходимости ряда $\sum_{k=1}^{\infty} t\left(c \lambda_{k}\right)$ получим сходимость ряда $\sum_{k=1}^{\infty}\left(\omega\left(t\left(c \lambda_{k}\right)\right) / \lambda_{k}\right)$. По лемме 2 из сходимости ряда $\sum_{k=1}^{\infty}\left(\omega\left(t\left(c \lambda_{k}\right)\right) / \lambda_{k}\right)$ следует сходимость ряда $\sum_{k=1}^{\infty} t\left(c \lambda_{k}\right)$. По теореме 1 получаем утверждение теоремы 2 .

ДоКАЗАТЕЛЬСТво ТЕОРемы 3. 1) Пусть в окрестности нуля $G_{1}\left(\omega_{+}^{\prime}(t)\right) \leqslant t$. Покажем, что $\omega(t) / t \rightarrow \infty$ при $t \rightarrow 0$. Допустим обратное. Тогда существует $M>0$ такое, что $\omega(t) / t \leqslant M$ при всех $t>0$, поскольку $\omega(t) / t$ - невозрастающая функция в силу вогнутости $\omega$. Так как $\omega_{+}^{\prime}(t) \leqslant \omega(t) / t$, то $\omega_{+}^{\prime}(t) \leqslant M$ при $t>0$. Поскольку $G_{1}(z)$ - невозрастающая функция, то $G_{1}\left(\omega_{+}^{\prime}(t)\right) \geqslant G_{1}(M)>0$ при всех $t>0$. Это противоречит предположению $G_{1}\left(\omega_{+}^{\prime}(t)\right) \leqslant t$ для малых $t$. Значит, $\lim _{t \rightarrow 0}(\omega(t) / t)=\infty$.

По определению $t(\lambda)$ имеем $\omega_{+}^{\prime}\left(t\left(c \lambda_{k}\right)\right) \leqslant c \lambda_{k}$ и, следовательно, $G_{1}\left(c \lambda_{k}\right) \leqslant$ $G_{1}\left(\omega_{+}^{\prime}\left(t\left(c \lambda_{k}\right)\right)\right)$. Пусть $H^{\omega} \subset \Lambda B V$. Тогда по теореме 2 найдется $c>0$ такое, что сходится ряд $\sum_{k=1}^{\infty} t\left(c \lambda_{k}\right)$. Так как $G_{1}\left(c \lambda_{k}\right) \leqslant G_{1}\left(\omega_{+}^{\prime}\left(t\left(c \lambda_{k}\right)\right)\right) \leqslant t\left(c \lambda_{k}\right)$ при больших $k$, то сходится ряд $\sum_{k=1}^{\infty} G_{1}\left(c \lambda_{k}\right)$.

$2)$ Пусть в окрестности нуля $t \leqslant G_{2}\left(\omega_{+}^{\prime}(t)\right)$. Зафиксируем $t$ из этой окрестности. Для $0<\tau<t$ имеем $\tau \leqslant G_{2}\left(\omega_{+}^{\prime}(\tau)\right)$ и $\omega_{+}^{\prime}(\tau) \geqslant \omega_{-}^{\prime}(t)$. Поскольку $G_{2}$ не возрастает, то $\tau \leqslant G_{2}\left(\omega_{-}^{\prime}(t)\right)$. Отсюда $t \leqslant G_{2}\left(\omega_{-}^{\prime}(t)\right)$.

По определению $t(\lambda)$ имеем $c \lambda_{k} \leqslant \omega_{-}^{\prime}\left(t\left(c \lambda_{k}\right)\right)$. Тогда $G_{2}\left(c \lambda_{k}\right) \geqslant G_{2}\left(\omega_{-}^{\prime}\left(t\left(c \lambda_{k}\right)\right)\right)$. Допустим, что при некотором положительном $c$ ряд $\sum_{k=1}^{\infty} G_{2}\left(c \lambda_{k}\right)$ сходится. Если справедливо $\lim _{t \rightarrow 0}(\omega(t) / t)<\infty$, то, как было отмечено, $H^{\omega} \subset \Lambda B V$. Пусть $\lim _{t \rightarrow 0}(\omega(t) / t)=\infty$. Так как $t\left(c \lambda_{k}\right) \leqslant G_{2}\left(\omega_{-}^{\prime}\left(t\left(c \lambda_{k}\right)\right)\right) \leqslant G_{2}\left(c \lambda_{k}\right)$ при больших $k$, то сходится ряд $\sum_{k=1}^{\infty} t\left(c \lambda_{k}\right)$. По теореме 2 получаем $H^{\omega} \subset \Lambda B V$. Теорема доказана. 
ДоКАЗАТЕЛЬСТво теоРемЫ 4. Обозначим $\tau=\varlimsup_{i m} \rightarrow \infty\left(\ln _{m} k / \xi_{k}\right)$. Докажем, что $\mu \geqslant \tau$. Известно [15], что если для невозрастающей последовательности положительных чисел $\left\{\gamma_{k}\right\}_{k=1}^{\infty}$ ряд $\sum_{k=1}^{\infty} \gamma_{k}$ сходится, то $\lim _{k \rightarrow \infty} k \gamma_{k}=0$. Если $\mu=\infty$, то неравенство $\mu \geqslant \tau$ выполняется. Пусть $\mu<\infty$. Тогда для любого $\varepsilon>0$ ряд $\sum_{k=1}^{\infty}\left(1 / \exp _{m}\left((\mu+\varepsilon) \xi_{k}\right)\right)$ сходится и, следовательно,

$$
\lim _{k \rightarrow \infty} \frac{k}{\exp _{m}\left((\mu+\varepsilon) \xi_{k}\right)}=0
$$

Поэтому для достаточно больших $k$ вьполняется $\exp _{m}\left((\mu+\varepsilon) \xi_{k}\right)>k$. Отсюда $(\mu+\varepsilon) \xi_{k}>\ln _{m} k$ и, значит, $\mu+\varepsilon \geqslant \tau$. Поскольку $\varepsilon$ произвольно, то $\mu \geqslant \tau$.

Докажем теперь, что $\mu \leqslant \tau$. В случае $\tau=\infty$ это неравенство выполняется. Пусть $\tau<\infty$. Будем рассматривать только те значения $k$, при которых $\ln k \geqslant 1$, $\ldots, \ln _{m} k \geqslant 1$. Возьмем произвольное $\varepsilon>0$. Имеем $\ln k^{1+\varepsilon}=(1+\varepsilon) \ln k$. Так как $\ln (1+\varepsilon)<\varepsilon$, то

$$
\ln _{2} k^{1+\varepsilon}=\ln _{2} k+\ln (1+\varepsilon) \leqslant \ln _{2} k+\varepsilon \leqslant \ln _{2} k+\varepsilon \ln _{2} k=(1+\varepsilon) \ln _{2} k .
$$

Аналогично,

$$
\ln _{3} k^{1+\varepsilon} \leqslant \ln _{3} k+\ln (1+\varepsilon) \leqslant \ln _{3} k+\varepsilon \leqslant \ln _{3} k+\varepsilon \ln _{3} k=(1+\varepsilon) \ln _{3} k .
$$

Продолжая логарифмировать, по индукции получаем $\ln _{m} k^{1+\varepsilon} \leqslant(1+\varepsilon) \ln _{m} k$.

Далее, по определению $\tau$ для достаточно больших $k$ имеем $\ln _{m} k \leqslant(\tau+\varepsilon) \xi_{k}$. Тогда $\ln _{m} k^{1+\varepsilon} \leqslant(1+\varepsilon) \ln _{m} k \leqslant(1+\varepsilon)(\tau+\varepsilon) \xi_{k}$. Поэтому

$$
k^{1+\varepsilon} \leqslant \exp _{m}\left((1+\varepsilon)(\tau+\varepsilon) \xi_{k}\right) .
$$

Поскольку ряд $\sum_{k=1}^{\infty}\left(1 / k^{1+\varepsilon}\right)$ сходится, то $\mu \leqslant(1+\varepsilon)(\tau+\varepsilon)$. В силу произвольности $\varepsilon$ получаем $\mu \leqslant \tau$. Следовательно, $\mu=\tau$. Теорема доказана.

Для доказательства теорем 5 и 6 нам потребуются леммы $3-8$.

ЛЕмма 3. Пусть для модуля непрерывности $\omega(t)=t u(x)$, где $x=\ln _{m-1}|\ln t|$ при мальх $t, m \in \mathbb{N}$, выполняется условие

$$
\frac{x u_{-}^{\prime}(x)}{u(x)}=\beta+\alpha(x), \quad \alpha(x)=o(1) \quad \text { npu } \quad x \rightarrow \infty, \quad \beta>0 .
$$

Тогда $\ln u(x)-\beta \ln x=o(\ln x)$ при $x \rightarrow \infty$.

ДоКАЗАТЕЛЬСТво. Проинтегрируем равенство $u_{-}^{\prime}(x) / u(x)=\beta / x+\alpha(x) / x$ от некоторого фиксированного $x_{0}$ до $x>x_{0}$. Получим

$$
\ln u(x)-\ln u\left(x_{0}\right)=\beta \ln x-\beta \ln x_{0}+\int_{x_{0}}^{x} \frac{\alpha(\tau)}{\tau} d \tau
$$

или

$$
\ln u(x)-\beta \ln x=\ln u\left(x_{0}\right)-\beta \ln x_{0}+\int_{x_{0}}^{x} \frac{\alpha(\tau)}{\tau} d \tau .
$$

Так как $\alpha(x)=o(1)$ при $x \rightarrow \infty$, то последний интеграл есть $o(\ln x)$ при $x \rightarrow \infty$, что и доказывает лемму. 
ЛЕМма 4. Если выполняется условие (1), то модуль непрерывности $\omega(t)$ удовлетворяет т-условию, $\lim _{t \rightarrow 0}(\omega(t) / t)=\infty, \ln \omega_{+}^{\prime}(t)-\ln u(x)=o(1)$ при $t \rightarrow 0 u \lim _{t \rightarrow 0}\left(\ln \omega_{+}^{\prime}(t) / \ln x\right)=\beta$.

ДокАЗАТЕльство. Продифференцируем равенство $\ln \omega(t)=\ln t+\ln u(x)$ по $t$. Найдем

$$
\frac{\omega_{+}^{\prime}(t)}{\omega(t)}=\frac{1}{t}+\frac{u_{-}^{\prime}(x)}{u(x)} x^{\prime}(t) .
$$

Если $m=1$, то $x^{\prime}(t)=-1 / t$. Если $m>1$, то

$$
x^{\prime}(t)=-\frac{1}{t|\ln t| \ln |\ln t| \cdots \ln _{m-2}|\ln t|}
$$

в окрестности нуля переменной $t$. Поэтому произведение $t x^{\prime}(t)$ ограничено в окрестности нуля. Тогда

$$
\frac{t \omega_{+}^{\prime}(t)}{\omega(t)}=1+\frac{u_{-}^{\prime}(x)}{u(x)} t x^{\prime}(t)=1+o(1) \text { при } \quad t \rightarrow 0
$$

так как $u_{-}^{\prime}(x) / u(x) \rightarrow 0$ при $x \rightarrow \infty$ в силу условия $(1)$. Отсюда следует равенство $\lim _{t \rightarrow 0}\left(t \omega_{+}^{\prime}(t) / \omega(t)\right)=1$. Поэтому $\omega(t)$ удовлетворяет $m$-условию.

В силу леммы 3 имеем $u(x) \rightarrow \infty$ при $x \rightarrow \infty$. Следовательно, $\omega(t) / t \rightarrow \infty$ при $t \rightarrow 0$. Далее, так как

$$
\frac{\omega_{+}^{\prime}(t)}{u(x)}=\frac{t \omega_{+}^{\prime}(t)}{\omega(t)} \rightarrow 1 \text { при } t \rightarrow 0
$$

то $\ln \omega_{+}^{\prime}(t)-\ln u(x)=o(1)$ при $t \rightarrow 0$. Тогда $\ln \omega_{+}^{\prime}(t) / \ln u(x) \rightarrow 1$ при $t \rightarrow 0$. Отсюда и из леммы 3 следует, что $\lim _{t \rightarrow 0}\left(\ln \omega_{+}^{\prime}(t) / \ln x\right)=\beta$. Лемма доказана.

ЛЕмма 5. Если выполняется условие (1), то для фиксированного $с>0$ справедливо $u(c x) \sim c^{\beta} u(x)$ при $x \rightarrow \infty$.

ДокАЗАТЕЛЬство. Пусть $c>1$. Проинтегрируем равенство $u_{-}^{\prime}(x) / u(x)=$ $\beta / x+\alpha(x) / x$ от $x>0$ до $c x$. Получим

$$
\ln \frac{u(c x)}{u(x)}=\beta \ln c+\int_{x}^{c x} \frac{\alpha(\tau)}{\tau} d \tau
$$

Оценим модуль интеграла

$$
\left|\int_{x}^{c x} \frac{\alpha(\tau)}{\tau} d \tau\right| \leqslant \sup _{\tau \in[x, c x]}|\alpha(\tau)| \int_{x}^{c x} \frac{d \tau}{\tau}=\sup _{\tau \in[x, c x]}|\alpha(\tau)| \ln c=o(1) \text { при } x \rightarrow \infty .
$$

Следовательно, $\lim _{x \rightarrow \infty} \ln (u(c x) / u(x))=\beta \ln c=\ln c^{\beta}$. Поэтому справедливо равенство $\lim _{x \rightarrow \infty}(u(c x) / u(x))=c^{\beta}$. Для случая $0<c<1$ доказательство аналогично, если интегрировать от $c x$ до $x$. Случай $c=1$ тривиален. Лемма доказана. 
ЛЕмма 6. Если выполняется условие (1), то функиия $x^{1+\beta} / u(x)$ возрасmaem при больиих $x$ u

$$
\lim _{x \rightarrow \infty} \frac{x^{1+\beta}}{u(x)}=\infty
$$

ДокАЗАТЕЛЬСТво. Найдем левую производную:

$$
\left(\frac{x^{1+\beta}}{u(x)}\right)_{-}^{\prime}=\frac{(1+\beta) x^{\beta} u(x)-x^{1+\beta} u_{-}^{\prime}(x)}{u^{2}(x)}=\frac{x^{\beta}}{u(x)}\left(1+\beta-\frac{x u_{-}^{\prime}(x)}{u(x)}\right)>0
$$

для больших $x$, поскольку по условию имеем $\beta-x u_{-}^{\prime}(x) / u(x) \rightarrow 0$ при $x \rightarrow \infty$.

В силу непрерывности функции $x^{1+\beta} / u(x)$ отсюда получаем, что при больших $x$ эта функция возрастает. По лемме 3 имеем $\ln u(x)-\beta \ln x=o(\ln x)$ при $x \rightarrow \infty$. Поэтому

$$
\ln \frac{x^{1+\beta}}{u(x)}=\ln x+\beta \ln x-\ln u(x)=\ln x+o(\ln x) \rightarrow \infty \text { при } x \rightarrow \infty .
$$

Значит, $\lim _{x \rightarrow \infty}\left(x^{1+\beta} / u(x)\right)=\infty$. Лемма доказана.

ЛЕмма 7. Если выполняется условие (1), то для функции

$$
H(t)=\ln \frac{x^{\beta} u\left(\omega_{+}^{\prime}(t)\right)}{\left(\omega_{+}^{\prime}(t)\right)^{1+\beta}}
$$

при $t \rightarrow 0$ справедливо

$$
H(t)=\int_{x}^{\omega_{+}^{\prime}(t)} \frac{\alpha(\tau)}{\tau} d \tau+o(1)
$$

ДокАЗАТЕЛьство. Проинтегрируем равенство $u_{-}^{\prime}(x) / u(x)=\beta / x+\alpha(x) / x$ от $x$ до $\omega_{+}^{\prime}(t)$. Получим

$$
\ln u\left(\omega_{+}^{\prime}(t)\right)-\ln u(x)=\beta \ln \omega_{+}^{\prime}(t)-\beta \ln x+\int_{x}^{\omega_{+}^{\prime}(t)} \frac{\alpha(\tau)}{\tau} d \tau
$$

или

$$
\ln u\left(\omega_{+}^{\prime}(t)\right)-\beta \ln \omega_{+}^{\prime}(t)-\ln u(x)+\beta \ln x=\int_{x}^{\omega_{+}^{\prime}(t)} \frac{\alpha(\tau)}{\tau} d \tau .
$$

Запишем $H(t)$ в следуюшем виде

$$
\begin{aligned}
H(t) & =\beta \ln x+\ln u\left(\omega_{+}^{\prime}(t)\right)-\ln \omega_{+}^{\prime}(t)-\beta \ln \omega_{+}^{\prime}(t) \\
& =\left(\ln u\left(\omega_{+}^{\prime}(t)\right)-\beta \ln \omega_{+}^{\prime}(t)-\ln u(x)+\beta \ln x\right)+\left(\ln u(x)-\ln \omega_{+}^{\prime}(t)\right) \\
& =\int_{x}^{\omega_{+}^{\prime}{ }^{(t)}} \frac{\alpha(\tau)}{\tau} d \tau-\left(\ln \omega_{+}^{\prime}(t)-\ln u(x)\right) .
\end{aligned}
$$

По лемме 4 имеем $\ln \omega_{+}^{\prime}(t)-\ln u(x)=o(1)$ при $t \rightarrow 0$. Отсюда следует утверждение леммы 7 . 
ЛЕмма 8. Если выполняется условие (1) и функиия

$$
H(t)=\ln \frac{x^{\beta} u\left(\omega_{+}^{\prime}(t)\right)}{\left(\omega_{+}^{\prime}(t)\right)^{1+\beta}}
$$

ограничена в окрестности нуля переменной $t$, то для вложения $H^{\omega} \subset \Lambda B V$ необходимо и достаточно, чтобы при некотором с $>0$ сходился ряд

$$
\sum_{k=1}^{\infty}\left(\exp _{m} \frac{c \lambda_{k}^{(1+\beta) / \beta}}{\left(u\left(\lambda_{k}\right)\right)^{1 / \beta}}\right)^{-1} .
$$

ДоКАЗАТЕЛЬСТВо. Из условия леммы следует, что найдутся положительные числа $A$ и $B$ такие, что в окрестности нуля переменной $t$ верно неравенство

$$
B \frac{\left(\omega_{+}^{\prime}(t)\right)^{1+\beta}}{u\left(\omega_{+}^{\prime}(t)\right)} \leqslant x^{\beta} \leqslant A \frac{\left(\omega_{+}^{\prime}(t)\right)^{1+\beta}}{u\left(\omega_{+}^{\prime}(t)\right)}
$$

и, следовательно,

$$
B^{1 / \beta} \frac{\left(\omega_{+}^{\prime}(t)\right)^{(1+\beta) / \beta}}{\left(u\left(\omega_{+}^{\prime}(t)\right)\right)^{1 / \beta}} \leqslant x \leqslant A^{1 / \beta} \frac{\left(\omega_{+}^{\prime}(t)\right)^{(1+\beta) / \beta}}{\left(u\left(\omega_{+}^{\prime}(t)\right)\right)^{1 / \beta}} .
$$

Поэтому в окрестности нуля переменной $t$ имеем

$$
\left(\exp _{m} A^{1 / \beta} \frac{\left(\omega_{+}^{\prime}(t)\right)^{(1+\beta) / \beta}}{\left(u\left(\omega_{+}^{\prime}(t)\right)\right)^{1 / \beta}}\right)^{-1} \leqslant t \leqslant\left(\exp _{m} B^{1 / \beta} \frac{\left(\omega_{+}^{\prime}(t)\right)^{(1+\beta) / \beta}}{\left(u\left(\omega_{+}^{\prime}(t)\right)\right)^{1 / \beta}}\right)^{-1}
$$

Если взять достаточно большое $z_{0}$, то при $z \geqslant z_{0}$ функции

$$
G_{1}(z)=\left(\exp _{m} A^{1 / \beta} \frac{z^{(1+\beta) / \beta}}{(u(z))^{1 / \beta}}\right)^{-1}, \quad G_{2}(z)=\left(\exp _{m} B^{1 / \beta} \frac{z^{(1+\beta) / \beta}}{(u(z))^{1 / \beta}}\right)^{-1}
$$

имеют смысл и по лемме 6 убывают. Продолжим их на $(0, \infty)$ так, чтобы они оставались невозрастающими. Мы доказали, что $G_{1}\left(\omega_{+}^{\prime}(t)\right) \leqslant t \leqslant G_{2}\left(\omega_{+}^{\prime}(t)\right)$ при малых $t$.

Пусть ряд (2) сходится при некотором $c_{0}>0$. Будем искать $c>0$ таким, чтобы при больших $k$ выполнялось неравенство

$$
B^{1 / \beta} \frac{\left(c \lambda_{k}\right)^{(1+\beta) / \beta}}{\left(u\left(c \lambda_{k}\right)\right)^{1 / \beta}}>\frac{c_{0} \lambda_{k}^{(1+\beta) / \beta}}{\left(u\left(\lambda_{k}\right)\right)^{1 / \beta}},
$$

которое равносильно неравенству $\left(u\left(c \lambda_{k}\right)\right)^{1 / \beta}<B^{1 / \beta} c^{(1+\beta) / \beta}\left(u\left(\lambda_{k}\right)\right)^{1 / \beta} / c_{0}$. По лемме 5 для фиксированного $c>0$ и больших $k$ справедливо $u\left(c \lambda_{k}\right)<(2 c)^{\beta} u\left(\lambda_{k}\right)$, т.е. $\left(u\left(c \lambda_{k}\right)\right)^{1 / \beta}<2 c\left(u\left(\lambda_{k}\right)\right)^{1 / \beta}$. Значит, при $c$ таком, что $2<B^{1 / \beta} c^{1 / \beta} / c_{0}$, будем иметь

$$
B^{1 / \beta} \frac{\left(c \lambda_{k}\right)^{(1+\beta) / \beta}}{\left(u\left(c \lambda_{k}\right)\right)^{1 / \beta}}>\frac{c_{0} \lambda_{k}^{(1+\beta) / \beta}}{\left(u\left(\lambda_{k}\right)\right)^{1 / \beta}} .
$$


Тогда при таком $c$ сходится ряд $\sum_{k=1}^{\infty} G_{2}\left(c \lambda_{k}\right)$. Поскольку $\omega(t)$ удовлетворяет $m$-условию, то по теореме 3 получаем $H^{\omega} \subset \Lambda B V$.

Пусть теперь $H^{\omega} \subset \Lambda B V$. Тогда по теореме 3 при некотором $c_{1}>0$ сходится ряд $\sum_{k=1}^{\infty} G_{1}\left(c_{1} \lambda_{k}\right)$. При больших $k$ по лемме 5 имеем $u\left(c_{1} \lambda_{k}\right)>\left(c_{1} / 2\right)^{\beta} u\left(\lambda_{k}\right)$. Поэтому

$$
\frac{A^{1 / \beta} c_{1}^{(1+\beta) / \beta} \lambda_{k}^{(1+\beta) / \beta}}{\left(u\left(c_{1} \lambda_{k}\right)\right)^{1 / \beta}}<\frac{2 A^{1 / \beta} c_{1}^{1 / \beta} \lambda_{k}^{(1+\beta) / \beta}}{\left(u\left(\lambda_{k}\right)\right)^{1 / \beta}}
$$

при больших $k$. Значит, при $c>2 A^{1 / \beta} c_{1}^{1 / \beta}$ для больших $k$ вьполняется неравенство

$$
G_{1}\left(c_{1} \lambda_{k}\right) \geqslant\left(\exp _{m} \frac{c \lambda_{k}^{(1+\beta) / \beta}}{\left(u\left(\lambda_{k}\right)\right)^{1 / \beta}}\right)^{-1}
$$

Поэтому сходится ряд

$$
\sum_{k=1}^{\infty}\left(\exp _{m} \frac{c \lambda_{k}^{(1+\beta) / \beta}}{\left(u\left(\lambda_{k}\right)\right)^{1 / \beta}}\right)^{-1}
$$

Лемма 8 доказана.

ДОКАЗАТЕЛЬСТво ТЕОРЕмЫ 5. Пусть $x u_{-}^{\prime}(x) / u(x)=\beta+\alpha(x)$, где $\alpha(x)=$ $O(1 / \ln x)$ при $x \rightarrow \infty, \beta>0, \beta \neq 1$. Тогда при больших $x$ и некотором $M>0$ имеем $|\alpha(x)| \leqslant M / \ln x$. Так как $\lim _{t \rightarrow 0}(\omega(t) / t)=\infty$, то $\omega_{+}^{\prime}(t) \rightarrow \infty$ при $t \rightarrow 0$. Поэтому при малых $t$

$$
\left|\int_{x}^{\omega_{+}^{\prime}(t)} \frac{\alpha(\tau)}{\tau} d \tau\right| \leqslant M\left|\int_{x}^{\omega_{+}^{\prime}(t)} \frac{d \tau}{\tau \ln \tau}\right|=M\left|\ln \frac{\ln \omega_{+}^{\prime}(t)}{\ln x}\right| .
$$

По лемме 4

$$
\lim _{t \rightarrow 0}\left|\ln \frac{\ln \omega_{+}^{\prime}(t)}{\ln x}\right|=|\ln \beta| .
$$

Поэтому интеграл ограничен в окрестности нуля переменной $t$.

По лемме 7

$$
H(t)=\int_{x}^{\omega_{+}^{\prime}(t)} \frac{\alpha(\tau)}{\tau} d \tau+o(1) \text { при } t \rightarrow 0 .
$$

Значит, функция

$$
H(t)=\ln \frac{x^{\beta} u\left(\omega_{+}^{\prime}(t)\right.}{\left(\omega_{+}^{\prime}(t)\right)^{1+\beta}}
$$

ограничена в окрестности нуля переменной $t$. Из леммы 8 следует заключение первой части теоремы.

Для доказательства второй части теоремы рассмотрим функции $\omega_{1}(t)=t u_{1}(x)$ и $\omega_{2}(t)=t u_{2}(x)$, которые определяются следуюшим образом. Зададим $\sigma>0$ и натуральное $r \geqslant 2$. Выберем $t_{0}>0$ так, чтобы для $x_{0}=x\left(t_{0}\right)$ выполнялось $\ln _{r} x_{0}>0$. Для $x \geqslant x_{0}$ положим

$$
u_{1}(x)=x^{\beta} \exp \left(-\int_{x_{0}}^{x} \frac{\left(\ln _{r} \tau\right)^{\sigma}}{\tau \ln \tau} d \tau\right), \quad u_{2}(x)=x^{\beta} \exp \left(\int_{x_{0}}^{x} \frac{\left(\ln _{r} \tau\right)^{\sigma}}{\tau \ln \tau} d \tau\right) .
$$


Мы опускаем длинные, но несложные выкладки, которые доказывают, что $\lim _{t \rightarrow 0} \omega_{i}(t)=0$ и что $\omega_{i}^{\prime}(t)>0, \omega_{i}^{\prime \prime}(t)<0, i=1,2$, при $0<t<t_{0}$, если $t_{0}$ достаточно мало. Кроме того,

$$
\frac{x u_{1}^{\prime}(x)}{u_{1}(x)}=\beta-\frac{\left(\ln _{r} x\right)^{\sigma}}{\ln x}, \quad \frac{x u_{2}^{\prime}(x)}{u_{2}(x)}=\beta+\frac{\left(\ln _{r} x\right)^{\sigma}}{\ln x} .
$$

Выберем положительное $t_{0} \leqslant 1$ так, чтобы кроме $\ln _{r} x_{0}>0$ вьполнялись неравенства $\omega_{i}^{\prime}(t)>0, \omega_{i}^{\prime \prime}(t)<0, i=1,2$, при $0<t<t_{0}$. Доопределим функции $\omega_{i}$, $i=1,2$, полагая $\omega_{i}(0)=0$ и $\omega_{i}(t)=\omega_{i}\left(t_{0}\right)$ для $t>t_{0}$. Тогда построенные функции являются вогнутьми модулями непрерывности на $[0, \infty)$.

Для модуля непрерьвности $\omega_{1}(t)$ составим функцию

$$
H_{1}(t)=\ln \frac{x^{\beta} u_{1}\left(\omega_{1}^{\prime}(t)\right)}{\left(\omega_{1}^{\prime}(t)\right)^{1+\beta}}
$$

По лемме 7 получим

$$
H_{1}(t)=-\int_{x}^{\omega_{1}^{\prime}(t)} \frac{\left(\ln _{r} \tau\right)^{\sigma}}{\tau \ln \tau} d \tau+o(1) \text { при } t \rightarrow 0
$$

Рассмотрим сначала случай $0<\beta<1$. По лемме 4

$$
\ln \omega_{1}^{\prime}(t)=\beta \ln x(1+o(1)) \text { при } t \rightarrow 0 .
$$

Тогда $\ln \omega_{1}^{\prime}(t)-\ln x=(\beta-1) \ln x(1+o(1))<0$ при больших $x$ (малых $\left.t\right)$. Отсюда $\omega_{1}^{\prime}(t)<x$ для больших $x$ (малых $t$ ).

Оценим $H_{1}(t)$ снизу в окрестности нуля, учитывая, что функция $\left(\ln _{r} x\right)^{\sigma} / \ln x$ убьвает при больших $x$. Из равенства (3) получим

$$
\begin{aligned}
H_{1}(t) & =\int_{\omega_{1}^{\prime}(t)}^{x} \frac{\left(\ln _{r} \tau\right)^{\sigma}}{\tau \ln \tau} d \tau+o(1) \geqslant \frac{\left(\ln _{r} x\right)^{\sigma}}{\ln x} \int_{\omega_{1}^{\prime}(t)}^{x} \frac{d \tau}{\tau}+o(1) \\
& =\frac{\left(\ln _{r} x\right)^{\sigma}}{\ln x}\left(\ln x-\ln \omega_{1}^{\prime}(t)\right)+o(1)=\left(\ln _{r} x\right)^{\sigma}\left(1-\frac{\ln \omega_{1}^{\prime}(t)}{\ln x}\right)+o(1)
\end{aligned}
$$

Так как по лемме $4 \lim _{x \rightarrow \infty}\left(\ln \omega_{1}^{\prime}(t) / \ln x\right)=\beta$, то

$$
\left(\ln _{r} x\right)^{\sigma}=\left(\ln _{r} \omega_{1}^{\prime}(t)\right)^{\sigma}(1+o(1)) \text { при } x \rightarrow \infty
$$

Тогда

$$
\left.H_{1}(t) \geqslant \frac{1-\beta}{2}\left(\ln _{r} \omega_{1}^{\prime}(t)\right)^{\sigma} \text { при больших } x \text { (при малых } t\right) .
$$

Из этого неравенства и определения $H_{1}(t)$ следует, что

$$
\frac{x^{\beta} u_{1}\left(\omega_{1}^{\prime}(t)\right)}{\left(\omega_{1}^{\prime}(t)\right)^{1+\beta}} \geqslant \exp \left(\frac{1-\beta}{2}\left(\ln _{r} \omega_{1}^{\prime}(t)\right)^{\sigma}\right)
$$


Отсюда

$$
x \geqslant \frac{\left(\omega_{1}^{\prime}(t)\right)^{(1+\beta) / \beta}}{\left(u_{1}\left(\omega_{1}^{\prime}(t)\right)\right)^{1 / \beta}} \exp \left(\frac{1-\beta}{2 \beta}\left(\ln _{r} \omega_{1}^{\prime}(t)\right)^{\sigma}\right) .
$$

Для достаточно больших $k$ обозначим $\xi_{k}=\ln _{m} k / \ln _{m+r} k$. Значение $\xi_{k}$ при больших $k$ возрастает с возрастанием $k$ и $\xi_{k} \rightarrow \infty$ при $k \rightarrow \infty$. Для больших $k$ определим $\lambda_{k}$

$$
\frac{\lambda_{k}^{(1+\beta) / \beta}}{\left(u_{1}\left(\lambda_{k}\right)\right)^{1 / \beta}}=\xi_{k}
$$

В силу леммы 6 непрерьвная функция $x^{1+\beta} / u_{1}(x)$ возрастает при больших $x$ и стремится к бесконечности при $x \rightarrow \infty$. Поэтому при больших $k$ значения $\lambda_{k}$ определены и возрастают к бесконечности при $k \rightarrow \infty$. Для начальных $k$ положительные $\lambda_{k}$ зададим произвольно, сохраняя монотонность последовательности $\left\{\lambda_{k}\right\}_{k=1}^{\infty}$. Имеем $\varlimsup_{k \rightarrow \infty}\left(\ln _{m} k / \xi_{k}\right)=\varlimsup_{\lim _{k \rightarrow \infty}} \ln _{m+r} k=\infty$. Значит, по теореме 4 при всех $c>0$ ряд

$$
\sum_{k=1}^{\infty}\left(\exp _{m} \frac{c \lambda_{k}^{(1+\beta) / \beta}}{\left(u_{1}\left(\lambda_{k}\right)\right)^{1 / \beta}}\right)^{-1}
$$

расходится.

Оценим $\ln \lambda_{k}$. Для этого прологарифмируем равенство, определяющее $\lambda_{k}$. Получим

$$
\frac{1+\beta}{\beta} \ln \lambda_{k}-\frac{1}{\beta} \ln u_{1}\left(\lambda_{k}\right)=\ln \xi_{k} .
$$

По лемме 3 при $k \rightarrow \infty$ выполняется $\ln u_{1}\left(\lambda_{k}\right)=\beta \ln \lambda_{k}(1+o(1))$. Поэтому

$$
\ln \xi_{k}=\frac{1+\beta}{\beta} \ln \lambda_{k}-\ln \lambda_{k}(1+o(1)) .
$$

Значит, при $k \rightarrow \infty$

$$
\ln \lambda_{k}=\beta \ln \xi_{k}(1+o(1))=\beta \ln \frac{\ln _{m} k}{\ln _{m+r} k}(1+o(1))=\beta \ln _{m+1} k(1+o(1)) .
$$

Отсюда, в частности, следует, что при больших $k \ln \lambda_{k}<\ln k$, т.е. $\lambda_{k}<k$. Значит, $\sum_{k=1}^{\infty}\left(1 / \lambda_{k}\right)=\infty$.

Далее, $\ln \ln \lambda_{k}=\ln \beta+\ln _{m+2} k+o(1)=\ln _{m+2} k(1+o(1))$ при $k \rightarrow \infty$. Отсюда $\ln _{r} \lambda_{k}=\ln _{m+r} k(1+o(1)), k \rightarrow \infty$. Из (4), учитывая, что по определению $\omega_{1}^{\prime}\left(t\left(c \lambda_{k}\right)\right)=c \lambda_{k}$, при больших $k$ получим

$$
\begin{aligned}
x\left(t\left(\lambda_{k}\right)\right) & \geqslant \frac{\lambda_{k}^{(1+\beta) / \beta}}{\left(u_{1}\left(\lambda_{k}\right)\right)^{1 / \beta}} \exp \left(\frac{1-\beta}{2 \beta}\left(\ln _{r} \lambda_{k}\right)^{\sigma}\right) \geqslant \xi_{k} \exp \left(\frac{1-\beta}{4 \beta}\left(\ln _{m+r} k\right)^{\sigma}\right) \\
& =\frac{\ln _{m} k}{\ln _{m+r} k} \exp \left(\frac{1-\beta}{4 \beta}\left(\ln _{m+r} k\right)^{\sigma}\right) .
\end{aligned}
$$

Поскольку

$$
\frac{1}{y} \exp \left(\frac{1-\beta}{4 \beta} y^{\sigma}\right) \rightarrow \infty \text { при } y \rightarrow \infty
$$


то при достаточно больших $k$ выполняется неравенство $x\left(t\left(\lambda_{k}\right)\right) \geqslant 2 \ln _{m} k \geqslant$ $\ln _{m} k^{2}$. Значит, $t\left(\lambda_{k}\right)=1 / \exp _{m}\left(x\left(t\left(\lambda_{k}\right)\right)\right) \leqslant 1 / k^{2}$ при больших $k$. Поэтому ряд $\sum_{k=1}^{\infty} t\left(\lambda_{k}\right)$ сходится. Так как по лемме 4 модуль непрерывности $\omega_{1}(t)$ удовлетворяет $m$-условию и $\lim _{t \rightarrow 0}\left(\omega_{1}(t) / t\right)=\infty$, то по теореме 2 , выбрав $c=1$, получим $H^{\omega} \subset \Lambda B V$.

Пусть теперь $\beta>1$. По лемме 4 имеем $\ln \omega_{1}^{\prime}(t)=\beta \ln x(1+o(1))$ при $t \rightarrow 0$. Тогда $\ln \omega_{1}^{\prime}(t)-\ln x=(\beta-1) \ln x(1+o(1))>0$ при больших $x$ (малых $\left.t\right)$. Отсюда $\omega_{1}^{\prime}(t)>x$ для больших $x$ (малых $t$ ). Оценим в равенстве $(3) H_{1}(t)$ сверху в окрестности нуля, учитывая, что функция $\left(\ln _{r} x\right)^{\sigma} / \ln x$ убывает при больших $x$. Из равенства (3) при больших $x$ (малых $t$ ) получим

$$
\begin{aligned}
H_{1}(t) & =-\int_{x}^{\omega_{1}^{\prime}(t)} \frac{\left(\ln _{r} \tau\right)^{\sigma}}{\tau \ln \tau} d \tau+o(1) \leqslant-\frac{\left(\ln _{r} x\right)^{\sigma}}{\ln x} \int_{x}^{\omega_{1}^{\prime}(t)} \frac{d \tau}{\tau}+o(1) \\
& =-\frac{\left(\ln _{r} x\right)^{\sigma}}{\ln x}\left(\ln \omega_{1}^{\prime}(t)-\ln x\right)+o(1)=-\left(\ln _{r} x\right)^{\sigma}\left(\frac{\ln \omega_{1}^{\prime}(t)}{\ln x}-1\right)+o(1) .
\end{aligned}
$$

Рассуждения, аналогичные тем, которые были проведены для случая $0<\beta<1$, при малых $t$ приводят к неравенству

$$
H_{1}(t) \leqslant-\frac{\beta-1}{2}\left(\ln _{r} \omega_{1}^{\prime}(t)\right)^{\sigma}
$$

Тогда

$$
x \leqslant \frac{\left(\omega_{1}^{\prime}(t)\right)^{(1+\beta) / \beta}}{\left(u_{1}\left(\omega_{1}^{\prime}(t)\right)\right)^{1 / \beta}} \exp \left(-\frac{\beta-1}{2 \beta}\left(\ln _{r} \omega_{1}^{\prime}(t)\right)^{\sigma}\right) .
$$

Не меняя обозначений, зададим теперь $\xi_{k}$ по-другому, а именно $\xi_{k}=\ln _{m} k$. Как и в случае $0<\beta<1$, определим $\lambda_{k}$ равенствами

$$
\frac{\lambda_{k}^{(1+\beta) / \beta}}{\left(u_{1}\left(\lambda_{k}\right)\right)^{1 / \beta}}=\xi_{k}
$$

Сушествование $\lambda_{k}$ и их возрастание при больших $k$, неравенство $\lambda_{k}<k$ и равенство $\ln _{r} \lambda_{k}=\ln _{m+r} k(1+o(1))$ при $k \rightarrow \infty$ доказываются так же, как в случае $0<\beta<1$. Поэтому снова ряд $\sum_{k=1}^{\infty}\left(1 / \lambda_{k}\right)$ расходится. Для любого $c>0$ из последнего равенства следует, что $\ln _{r}\left(c \lambda_{k}\right)=\ln _{m+r} k(1+o(1))$ при $k \rightarrow \infty$.

Докажем, что для каждого $c>0$ ряд $\sum_{k=1}^{\infty} t\left(c \lambda_{k}\right)$ расходится. Зафиксируем $c$. Подставим $t=t\left(c \lambda_{k}\right)$ в $(5)$, учитьвая, что $c \lambda_{k}=\omega_{1}^{\prime}\left(t\left(c \lambda_{k}\right)\right)$. Получим

$$
x\left(t\left(c \lambda_{k}\right)\right) \leqslant \frac{\left(c \lambda_{k}\right)^{(1+\beta) / \beta}}{\left(u_{1}\left(c \lambda_{k}\right)\right)^{1 / \beta}} \exp \left(-\frac{\beta-1}{2 \beta}\left(\ln _{r}\left(c \lambda_{k}\right)\right)^{\sigma}\right)
$$

Если $k$ достаточно большое, то в силу $\ln _{r}\left(c \lambda_{k}\right) \sim \ln _{m+r} k$ и леммы 5

$$
\left(\ln _{r}\left(c \lambda_{k}\right)\right)^{\sigma} \geqslant \frac{1}{2}\left(\ln _{m+r} k\right)^{\sigma}, \quad\left(u_{1}\left(c \lambda_{k}\right)\right)^{1 / \beta} \geqslant \frac{1}{2} c\left(u_{1}\left(\lambda_{k}\right)\right)^{1 / \beta}
$$


Тогда

$$
\begin{aligned}
x\left(t\left(c \lambda_{k}\right)\right) & \leqslant \frac{c^{(1+\beta) / \beta} \lambda_{k}^{(1+\beta) / \beta}}{(1 / 2) c\left(u_{1}\left(\lambda_{k}\right)\right)^{1 / \beta}} \exp \left(-\frac{\beta-1}{4 \beta}\left(\ln _{m+r} k\right)^{\sigma}\right) \\
& =2 c^{1 / \beta} \xi_{k} \exp \left(-\frac{\beta-1}{4 \beta}\left(\ln _{m+r} k\right)^{\sigma}\right) \\
& =2 c^{1 / \beta} \ln _{m} k \exp \left(-\frac{\beta-1}{4 \beta}\left(\ln _{m+r} k\right)^{\sigma}\right) .
\end{aligned}
$$

Поскольку $\exp (-(\beta-1) y /(4 \beta)) \rightarrow 0$ при $y \rightarrow \infty$, то $x\left(t\left(c \lambda_{k}\right)\right) \leqslant \ln _{m} k$ при больших $k$. Тогда

$$
t\left(c \lambda_{k}\right)=\frac{1}{\exp _{m}\left(x\left(t\left(c \lambda_{k}\right)\right)\right)} \geqslant \frac{1}{k} .
$$

Следовательно, ряд $\sum_{k=1}^{\infty} t\left(c \lambda_{k}\right)$ расходится. По теореме 2 получим $H^{\omega} \not \subset \Lambda B V$.

$\mathrm{C}$ другой стороны, так как $\overline{\lim }_{k \rightarrow \infty}\left(\ln _{m} k / \xi_{k}\right)=1$, то по теореме 4 сушествует $c>0$ такое, что сходится ряд

$$
\sum_{k=1}^{\infty}\left(\exp _{m} \frac{c \lambda_{k}^{(1+\beta) / \beta}}{\left(u_{1}\left(\lambda_{k}\right)\right)^{1 / \beta}}\right)^{-1}=\sum_{k=1}^{\infty} \frac{1}{\exp _{m}\left(c \xi_{k}\right)}
$$

Для модуля непрерьвности $\omega_{2}(t)$ составим функцию

$$
H_{2}(t)=\ln \frac{x^{\beta} u_{2}\left(\omega_{2}^{\prime}(t)\right)}{\left(\omega_{2}^{\prime}(t)\right)^{1+\beta}}
$$

По лемме 7 получим

$$
H_{2}(t)=\int_{x}^{\omega_{2}^{\prime}(t)} \frac{\left(\ln _{r} \tau\right)^{\sigma}}{\tau \ln \tau} d \tau+o(1) \text { при } t \rightarrow 0
$$

Здесь по сравнению со случаем $H_{1}$ перед интегралом стоит противоположный знак. Теперь для $H_{2}(t)$ в случае $0<\beta<1$ надо провести рассуждения, аналогичные тем, которые были приведены для $H_{1}(t)$, но в случае $\beta>1$. Случай $\beta>1$ для $H_{2}(t)$ аналогичен случаю $0<\beta<1$ для $H_{1}(t)$.

Итак, если изменить порядок бесконечно малой, как указано во второй части теоремы, то для каждого $\beta>0, \beta \neq 1$, возможен случай, когда $H^{\omega} \subset \Lambda B V$, но ряд (2) расходится при всех $c>0$, и возможен случай, когда этот ряд сходится при некотором $c>0$, но $H^{\omega} \not \subset \Lambda B V$. Теорема доказана.

ДоКАЗАТЕЛЬСтво ТЕОРЕмЫ 6. Докажем первую часть теоремы. Пусть справедливо равенство $x u_{-}^{\prime}(x) / u(x)=1+\alpha(x)$, где $\alpha(x)=O(1 / \sqrt{\ln x})$ при $x \rightarrow \infty$. Здесь $\beta=1$. Проинтегрируем $u_{-}^{\prime}(x) / u(x)=1 / x+\alpha(x) / x$ от $x_{0}$ до $x \geqslant x_{0}$. Получим

$$
\ln u(x)-\ln u\left(x_{0}\right)=\ln x-\ln x_{0}+\int_{x_{0}}^{x} \frac{\alpha(\tau)}{\tau} d \tau .
$$


Поскольку при больших $x$ для некоторого $M>0$ выполняется $|\alpha(x)| \leqslant M / \sqrt{\ln x}$, TO

$$
\begin{aligned}
& |\ln u(x)-\ln x| \leqslant\left|\ln u\left(x_{0}\right)-\ln x_{0}\right|+\left|\int_{x_{0}}^{x} \frac{\alpha(\tau)}{\tau} d \tau\right| \\
& \leqslant\left|\ln u\left(x_{0}\right)-\ln x_{0}\right|+M \int_{x_{0}}^{x} \frac{d \tau}{\tau \sqrt{\ln \tau}}=\left|\ln u\left(x_{0}\right)-\ln x_{0}\right|+2 M\left(\sqrt{\ln x}-\sqrt{\ln x_{0}}\right) .
\end{aligned}
$$

Поэтому $\ln u(x)-\ln x=O(\sqrt{\ln x})$ при $x \rightarrow \infty$.

По лемме $4 \ln \omega_{+}^{\prime}(t)-\ln u(x)=o(1)$ при $t \rightarrow 0$. Тогда $\ln \omega_{+}^{\prime}(t)-\ln x=O(\sqrt{\ln x})$ при $x \rightarrow \infty$. По лемме 7 при $t \rightarrow 0$

$$
H(t)=\ln \frac{x^{\beta} u\left(\omega_{+}^{\prime}(t)\right)}{\left(\omega_{+}^{\prime}(t)\right)^{1+\beta}}=\int_{x}^{\omega_{+}^{\prime}(t)} \frac{\alpha(\tau)}{\tau} d \tau+o(1)
$$

Следовательно,

$$
\begin{aligned}
|H(t)| & \leqslant\left|\int_{x}^{\omega_{+}^{\prime}(t)} \frac{\alpha(\tau)}{\tau} d \tau\right|+o(1) \leqslant M\left|\int_{x}^{\omega_{+}^{\prime}(t)} \frac{d \tau}{\tau \sqrt{\ln \tau}}\right|+o(1) \\
& =2 M\left|\sqrt{\ln \omega_{+}^{\prime}(t)}-\sqrt{\ln x}\right|+o(1)=2 M \frac{\left|\ln \omega_{+}^{\prime}(t)-\ln x\right|}{\sqrt{\ln \omega_{+}^{\prime}(t)}+\sqrt{\ln x}}+o(1) \\
& \leqslant 2 M \frac{\left|\ln \omega_{+}^{\prime}(t)-\ln x\right|}{\sqrt{\ln x}}+o(1) .
\end{aligned}
$$

Поскольку $\ln \omega_{+}^{\prime}(t)-\ln x=O(\sqrt{\ln x})$ при $x \rightarrow \infty$, то функция $H(t)$ ограничена в окрестности нуля переменной $t$. Отсюда по лемме 8 получим, что $H^{\omega} \subset \Lambda B V$ тогда и только тогда, когда при некотором $c>0$ сходится ряд

$$
\sum_{k=1}^{\infty}\left(\exp _{m} \frac{c \lambda_{k}^{2}}{u\left(\lambda_{k}\right)}\right)^{-1}
$$

Первая часть теоремы доказана.

Докажем вторую часть теоремы. Зададим $\sigma>0$ и натуральное $r \geqslant 2$. Рассмотрим функцию $\omega(t)=t u(x), u(x)$ при больших $x$ (малых $t$ ) задается формулой

$$
u(x)=x \exp \left(-\int_{x_{0}}^{x} \frac{\left(\ln _{r} \tau\right)^{\sigma}}{\tau \sqrt{\ln \tau}} d \tau\right)
$$

где $x \geqslant x_{0}=x\left(t_{0}\right), x_{0}$ достаточно большое ( $t_{0}$ достаточно малое), чтобы выполнялось неравенство $\ln _{r} x_{0}>0$. Мы не приводим громоздкие, но не вызывающие существенных затруднений выкладки, которые доказывают, что $\lim _{t \rightarrow 0} \omega(t)=0$ и что $\omega^{\prime}(t)>0, \omega^{\prime \prime}(t)<0$ при $0<t \leqslant t_{0}$, если $t_{0}$ достаточно мало. Кроме того,

$$
\frac{x u^{\prime}(x)}{u(x)}=1-\frac{\left(\ln _{r} x\right)^{\sigma}}{\sqrt{\ln x}}
$$


Функция $\omega$ на полупрямой $[0, \infty)$ строится так же, как функции $\omega_{1}, \omega_{2}$ в доказательстве теоремы 5 . Тогда $\omega$ - вогнутый модуль непрерывности на $[0, \infty)$.

Из определения $u(x)$ получим

$$
\ln u(x)=\ln x-\int_{x_{0}}^{x} \frac{\left(\ln _{r} \tau\right)^{\sigma}}{\tau \sqrt{\ln \tau}} d \tau
$$

По лемме 4 имеем $\ln \omega^{\prime}(t)-\ln u(x)=o(1)$ при $t \rightarrow 0$. Поэтому при $t \rightarrow 0$

$$
\ln \omega^{\prime}(t)=\ln u(x)+o(1)=\ln x-\int_{x_{0}}^{x} \frac{\left(\ln _{r} \tau\right)^{\sigma}}{\tau \sqrt{\ln \tau}} d \tau+o(1)
$$

Значит, при $t \rightarrow 0$

$$
\ln x-\ln \omega^{\prime}(t)=\int_{x_{0}}^{x} \frac{\left(\ln _{r} \tau\right)^{\sigma}}{\tau \sqrt{\ln \tau}} d \tau+o(1) .
$$

Будем считать, что $x_{0}$ достаточно большое, чтобы при $\tau \geqslant x_{0}$ выполнялось неравенство $\left(\ln _{r} \tau\right)^{\sigma} \geqslant 5$.

Так как

$$
\int_{x_{0}}^{x} \frac{\left(\ln _{r} \tau\right)^{\sigma}}{\tau \sqrt{\ln \tau}} d \tau \geqslant 5 \int_{x_{0}}^{x} \frac{d \tau}{\tau \sqrt{\ln \tau}}=10\left(\sqrt{\ln x}-\sqrt{\ln x_{0}}\right)
$$

то при больших $x$ справедливо $\ln x-\ln \omega^{\prime}(t) \geqslant 5 \sqrt{\ln x}$. Отсюда, в частности, следует, что $x>\omega^{\prime}(t)$. По лемме 7 для $\beta=1$ получим, что для функции

$$
H(t)=\ln \frac{x u\left(\omega^{\prime}(t)\right)}{\left(\omega^{\prime}(t)\right)^{2}}
$$

вьполняется следующее

$$
H(t)=-\int_{x}^{\omega^{\prime}(t)} \frac{\left(\ln _{r} \tau\right)^{\sigma}}{\tau \sqrt{\ln \tau}} d \tau+o(1)=\int_{\omega^{\prime}(t)}^{x} \frac{\left(\ln _{r} \tau\right)^{\sigma}}{\tau \sqrt{\ln \tau}} d \tau+o(1) \text { при } t \rightarrow 0
$$

Поскольку при больших $x$ функция $\left(\ln _{r} x\right)^{\sigma} / \sqrt{\ln x}$ убывает, то в окрестности нуля переменной $t$ получаем

$$
\begin{aligned}
H(t) & \geqslant \frac{\left(\ln _{r} x\right)^{\sigma}}{\sqrt{\ln x}} \int_{\omega^{\prime}(t)}^{x} \frac{d \tau}{\tau}+o(1)=\frac{\left(\ln _{r} x\right)^{\sigma}}{\sqrt{\ln x}}\left(\ln x-\ln \omega^{\prime}(t)\right)+o(1) \\
& \geqslant \frac{\left(\ln _{r} x\right)^{\sigma}}{\sqrt{\ln x}} 5 \sqrt{\ln x}+o(1) \geqslant 4\left(\ln _{r} x\right)^{\sigma} .
\end{aligned}
$$

По лемме 4 для $\beta=1$ имеем $\ln \omega^{\prime}(t)=\ln x(1+o(1))$ при $t \rightarrow 0$. Отсюда $\left(\ln _{r} x\right)^{\sigma}=$ $\left(\ln _{r} \omega^{\prime}(t)\right)^{\sigma}(1+o(1))$ при $t \rightarrow 0$. Поэтому при малых $t$ получаем, что $H(t) \geqslant$ $3\left(\ln _{r} \omega^{\prime}(t)\right)^{\sigma}$, т.е. $x u\left(\omega^{\prime}(t)\right) /\left(\omega^{\prime}(t)\right)^{2} \geqslant 3\left(\ln _{r} \omega^{\prime}(t)\right)^{\sigma}$. Значит,

$$
x \geqslant \frac{3\left(\ln _{r} \omega^{\prime}(t)\right)^{\sigma}\left(\omega^{\prime}(t)\right)^{2}}{u\left(\omega^{\prime}(t)\right)} .
$$


Положим $\xi_{k}=\ln _{m} k /\left(\ln _{m+r} k\right)^{\sigma}$ при достаточно больших $k$. Значение $\xi_{k}$ возрастает к бесконечности с возрастанием $k$. При больших $k$ определим $\lambda_{k}$ равенствами

$$
\frac{\lambda_{k}^{2}}{u\left(\lambda_{k}\right)}=\xi_{k}
$$

По лемме 6 для $\beta=1$ получим, что при достаточно больших $k$ числа $\lambda_{k}$ сушествуют и возрастают к бесконечности с возрастанием $k$. Для начальных значений $k$ зададим $\lambda_{k}>0$ произвольным образом, сохраняя монотонность последовательности $\left\{\lambda_{k}\right\}_{k=1}^{\infty}$.

Так как $\overline{\lim }_{k \rightarrow \infty}\left(\ln _{m} k / \xi_{k}\right)=\varlimsup_{k \rightarrow \infty}\left(\ln _{m+r} k\right)^{\sigma}=\infty$, то из теоремы 4 следует, что ряд

$$
\sum_{k=1}^{\infty}\left(\exp _{m} \frac{c \lambda_{k}^{2}}{u\left(\lambda_{k}\right)}\right)^{-1}=\sum_{k=1}^{\infty} \frac{1}{\exp _{m}\left(c \xi_{k}\right)}
$$

расходится при всех $c>0$.

Оценим $\ln \lambda_{k}$. Для этого прологарифмируем равенство, определяющее $\lambda_{k}$. Получим $2 \ln \lambda_{k}-\ln u\left(\lambda_{k}\right)=\ln \xi_{k}$. По лемме 3 для $\beta=1$ при $k \rightarrow \infty$ имеем $\ln u\left(\lambda_{k}\right)=\ln \lambda_{k}(1+o(1))$. Поэтому $\ln \xi_{k}=2 \ln \lambda_{k}-\ln \lambda_{k}(1+o(1))$. Значит,

$\ln \lambda_{k}=\ln \xi_{k}(1+o(1))=\ln \frac{\ln _{m} k}{\left(\ln _{m+r} k\right)^{\sigma}}(1+o(1))=\ln _{m+1} k(1+o(1))$ при $k \rightarrow \infty$.

Отсюда имеем $\ln \lambda_{k}<\ln k$ при больших $k$, т.е. $\lambda_{k}<k$. Значит, $\sum_{k=1}^{\infty}\left(1 / \lambda_{k}\right)=\infty$. Кроме того, $\left(\ln _{r} \lambda_{k}\right)^{\sigma}=\left(\ln _{m+r} k\right)^{\sigma}(1+o(1))$ при $k \rightarrow \infty$.

Подставим $t=t\left(\lambda_{k}\right)$ в неравенство (6), учитьвая, что $\omega^{\prime}\left(t\left(c \lambda_{k}\right)\right)=c \lambda_{k}$. При больших $k$ получим

$$
x\left(t\left(\lambda_{k}\right)\right) \geqslant \frac{3\left(\ln _{r} \lambda_{k}\right)^{\sigma} \lambda_{k}^{2}}{u\left(\lambda_{k}\right)} \geqslant 2\left(\ln _{m+r} k\right)^{\sigma} \xi_{k}=2 \ln _{m} k \geqslant \ln _{m} k^{2} .
$$

Значит, $t\left(\lambda_{k}\right)=1 / \exp _{m}\left(x\left(t\left(\lambda_{k}\right)\right)\right) \leqslant 1 / k^{2}$ при больших $k$. Поэтому ряд $\sum_{k=1}^{\infty} t\left(\lambda_{k}\right)$ сходится. Так как по лемме 4 модуль непрерывности $\omega(t)$ удовлетворяет $m$-условию и $\lim _{t \rightarrow 0}(\omega(t) / t)=\infty$, то отсюда по теореме 2 для $c=1$ получим $H^{\omega} \subset \Lambda B V$.

Итак, если изменить предположение о порядке бесконечно малой, как указано во второй части теоремы, то возможен случай, когда $H^{\omega} \subset \Lambda B V$, но ряд, указанный в теореме 6 , расходится при всех $c>0$.

Докажем третью часть теоремы. Пусть $\alpha(x)=x u_{-}^{\prime}(x) / u(x)-1=o(1)$ при $x \rightarrow \infty$ и $\alpha(x)$ не меняет знака при больших $x$. Интегрируя равенство $u_{-}^{\prime}(x) / u(x)=1 / x+\alpha(x) / x$ от $x_{0}$ до $x \geqslant x_{0}$, получим

$$
\ln u(x)-\ln x=\ln u\left(x_{0}\right)-\ln x_{0}+\int_{x_{0}}^{x} \frac{\alpha(\tau)}{\tau} d \tau
$$

Если $\alpha(x)=0$ почти всюду на промежутке $\left[x_{0}, \infty\right)$, то на этом промежутке $u(x)=$ $b x$ для некоторого $b>0$. Тогда $\alpha(x)=0$ всюду на $\left[x_{0}, \infty\right)$. Следовательно, из первой части теоремы 6 получим $H^{\omega} \subset \Lambda B V$. 
Пусть теперь для сколь угодно большого $x_{0}$ функция $\alpha(x)$ отлична от нуля на множестве положительной меры промежутка $\left[x_{0}, \infty\right)$. Зафиксируем $x_{0}$ такое, что $\alpha(x)$ не меняет знак на $\left[x_{0}, \infty\right)$. Если функцию $u(x)$ умножить на положительное число, то класс $H^{\omega}$ не изменится. При этом не изменится и функция $\alpha(x)$. Поэтому можно считать, что $u\left(x_{0}\right)=x_{0}$. Тогда $\ln u(x)-\ln x=\int_{x_{0}}^{x} \alpha(\tau) / \tau d \tau$. Докажем, что функция

$$
H(t)=\ln \frac{x u\left(\omega_{+}^{\prime}(t)\right)}{\left(\omega_{+}^{\prime}(t)\right)^{2}}
$$

ограничена снизу в окрестности нуля.

По лемме 4 имеем $\ln \omega_{+}^{\prime}(t)-\ln u(x)=o(1)$ при $t \rightarrow 0$. Тогда

$$
\ln \omega_{+}^{\prime}(t)-\ln x=\int_{x_{0}}^{x} \frac{\alpha(\tau)}{\tau} d \tau+o(1) \text { при } t \rightarrow 0
$$

Пусть $\alpha(x) \geqslant 0$ при $x \geqslant x_{0}$. Так как $\alpha(x)$ отлична от нуля на множестве положительной меры, то $\ln \omega_{+}^{\prime}(t)-\ln x=\int_{x_{0}}^{x} \alpha(\tau) / \tau d \tau+o(1) \geqslant 0$ при больших $x$. Поэтому $\omega_{+}^{\prime}(t) \geqslant x$. Аналогично, если $\alpha(x) \leqslant 0$ при $x \geqslant x_{0}$, то $\ln \omega_{+}^{\prime}(t)-\ln x \leqslant 0$ при больших $x$ и, значит, $\omega_{+}^{\prime}(t) \leqslant x$.

По лемме 7 имеем

$$
H(t)=\int_{x}^{\omega_{+}^{\prime}(t)} \frac{\alpha(\tau)}{\tau} d \tau+o(1) \text { при } t \rightarrow 0
$$

Следовательно, если $\alpha(x) \geqslant 0$ при $x \geqslant x_{0}$, то $H(t) \geqslant 0$ в окрестности нуля. Если $\alpha(x) \leqslant 0$ при $x \geqslant x_{0}$, то

$$
H(t)=\int_{x}^{\omega_{+}^{\prime}(t)} \frac{\alpha(\tau)}{\tau} d \tau+o(1)=-\int_{\omega_{+}^{\prime}(t)}^{x} \frac{\alpha(\tau)}{\tau} d \tau+o(1) \geqslant 0
$$

в окрестности нуля. Итак, функция $H(t)$ ограничена снизу в окрестности нуля. Из доказательства леммы 8 следует, что в случае $\beta=1$ для вложения $H^{\omega} \subset \Lambda B V$ достаточно, чтобы при некотором $c>0$ сходился ряд

$$
\sum_{k=1}^{\infty}\left(\exp _{m} \frac{c \lambda_{k}^{2}}{u\left(\lambda_{k}\right)}\right)^{-1}
$$

Теорема доказана. 
ДоКАЗАТЕЛЬСТво СлЕДСТВИЯ 1 . Класс $H^{\omega}$ не изменится, если будем считать, что в некоторой окрестности нуля переменной $t$

$$
u(x)=x^{\beta_{m}}(\ln x)^{\beta_{m+1}} \cdots\left(\ln _{n-m} x\right)^{\beta_{n}}, \quad \text { где } x=\ln _{m-1}|\ln t| .
$$

Дифференцируя $\ln u(x)$ по $x$, получаем

$$
\frac{u^{\prime}(x)}{u(x)}=\frac{\beta_{m}}{x}+\frac{\beta_{m+1}}{x \ln x}+\cdots+\frac{\beta_{n}}{x \ln x \cdots \ln _{n-m} x} .
$$

Отсюда $x u^{\prime}(x) / u(x)=\beta_{m}+O(1 / \ln x)$. Для $\beta \neq 1$ выполняется условие теоремы 5 , а для $\beta=1$ выполняется условие теоремы 6 . Отсюда следует утверждение следствия.

Используя лемму 6 и теорему 4, из теорем 5,6 и следствия 1 непосредственно получаем теоремы 7,8 и следствие 2 .

Автор выражает глубокую благодарность своему научному руководителю П. Л. Ульянову за постановку задач и внимание к работе.

\section{Список литературы}

1. Бари Н. К. Тригонометрические ряды. М.: ГИФМЛ, 1961.

2. Wiener $N$. The quadratic variation of a function and its Fourier coefficients // Mass. J. Math. 1924. V. 3. P. 72-94.

3. Marcinkiewicz J. On a class of functions and their Fourier series // C. R. Soc. Sci. Varsovie. 1934. V. 26. P. 71-77.

4. Young L.C. Sur une generalisation de la notion de variation de puissance $p$-ieme bornee au sens de M.Wiener, et sur la convergence des series de Fourier // C. R. Acad. Sci. Paris. 1937. V. 204. P. 470-472.

5. Salem R. Essais sur les series trigonometriques. Paris: Hermann, 1940.

6. Waterman $D$. On convergence of Fourier series of functions of generalized bounded variation // Studia Math. 1972. V. 44. № 2. P. 107-117.

7. Медведева М.В. О вложении классов $H^{\omega} / /$ Матем. заметки. 1998. Т. 64. №5. C. $713-719$

8. Белов $A$. C. Соотношения между различньми классами функций обобщенной ограниченной вариации // Доклады расширенных заседаний семинара Института им. И.Н. Векуа. Т. 3. № 2. Тбилиси, 1988. С. 11-14.

9. Бережной Е. И. Пространства функций обобщенной ограниченной вариации. Теоремы вложения. Оценки констант Лебега // Сиб. матем. журн. 1999. Т. 40. № 5. С. 997-1011.

10. Leindler L. A note on embedding of classes $H^{\omega} / /$ Anal. Math. 2001. V. 27. № 1. P. 71-76.

11. Медведева M. В. Условия вложений классов функций $H^{\omega}$ в классы функций ограниченной обобщенной вариации // Вестн. МГУ. Сер.1. Матем., мех. 2001. № 2. С. 60-64.

12. Ефимов А. В. Линейные методы приближения непрерьвњых периодических функций // Матем. сб. 1961. Т. 54. № 1. С. 51-90.

13. Красносельский М. А., Рутицкий Я. Б. Вьпуклые функции и пространства Орлича. М.: ГИФМЛ, 1958.

14. Натансон И. П. Конструктивная теория функций. М.: Гос. изд. тех.-теор. лит., 1949.

15. Маркушевич А. И. Теория аналитических функций. Т. 2. М.: Наука, 1968.

Московский государственный

Поступила в редакцию

университет им. М.В. Ломоносова

22.11 .2001 